\title{
Experiments on computer assisted optimization of the escherichia coli fermentation process using Optferm
}

\author{
Tânia Teixeira ${ }^{1}$ and Sérgio Deusdado ${ }^{2}$ \\ ${ }^{1}$ Superior Agrarian School, Polytechnic Institute of Bragança, Bragança, Portugal \\ ${ }^{2} \mathrm{CIMO}$ - Mountain Research Center, Polytechnic Institute of Bragança, Bragança, Portugal
}

\begin{abstract}
We used the software Optferm to model and optimize the bioprocess of escherichia coli fermentation, simulating the conditions of a fed-batch bioreactor. The results, obtained by employing different AI approaches, provided useful orientation for nutrient feed strategies for increased productivity. In conclusion, we believe in silico tools can perform a rapid and valid optimization of bioprocesses avoiding expensive experiments.
\end{abstract}

\section{Introduction}

During the last two decades, due to the rapid development of molecular biology research, the amount of genomic, proteomic, metabolomic and phylogenetic data has grown exponentially, forcing researchers to take advantage of computational tools to store, communicate and handle biological data. As a result, bioinformatics has emerged from this need to address the biodata management issues, as well as assisting the inference of knowledge that leads to functional understanding.

The need to include, adapt and maximize the productivity of bioprocesses in different biotechnological industries fostered the development of software that can assist in the optimization of biotechnological productions that rely on microorganisms. In this context, several programs have been developed, allowing in silico modeling, simulation and optimization of well known bioprocesses [1][2][3][4].

\section{Platform and Methods}

We studied the state of the art to evaluate the available tools in order to select the most appropriate one for the purpose of escherichia coli fermentation optimization [5][6]. The selected tool was Optferm, presented and described in [7]. The 
main reasons that support our choice were, firstly, the need to use a publicly available tool, (Opfterm is accessible at http://darwin.di.uminho.pt/optferm), besides that, Optferm offers a platform-independent, user-friendly, open-source and extensible environment for the simulation and optimization of fermentation processes.

OptFerm is a software platform that integrates a variety of AI algorithms, including artificial neural networks and evolutionary algorithms with the aim of helping the optimization of the biotechnological fermentation. It is an easy tool to use, modular, programmed in Java, which allows carrying out several tasks of simulation, optimization and parameter estimation with different conditions with respect to state variables, parameters, feed profiles, among others, used in fed-batch type bioreactors.

\section{Experimental work}

Attempting to test the applicability and efficiency of OptFerm, we decided to use escherichia coli fermentation model and simulation to gather the optimization results obtained from different IA algorithms and compare their performance and consistency. Finally, a comparative analysis was done in order to validate the used methodologies and assess the reliability of the results.

The model and the initial parameters used in this work were published in [6] and later incorporated in Optferm. Thus, the state variables used and the initial kinetic parameters are the result of experimental work developed in real conditions and are crucial to the quality of the obtained results.

The objective function established for the optimization was:

$$
J=\text { final } X^{\star} \text { final } W \text {-initial } X^{*} W \text { per time unit }
$$

, where $X$ represents the biomass concentration and $\mathrm{W}$ represents the weight of liquid inside the bioreactor.

Optferm is actually a beta version tool, so the experimental work was done in close contact with Orlando Rocha, from the developing team at the Minho University, which gently helped us a lot and corrected some software bugs we detected during our work with the tool.

\section{Results}

Tests were performed on a system based on an Intel Xeon ${ }^{\circledR}$ - E5405 2GHz CPU, with 2GB RAM, 4MB cache and using a 64-bit Linux operating system. On 
average, we had regularly more than $90 \%$ of processor availability to perform our tests.

We used three different algorithms, included in Optferm, to test the optimizations of the escherichia coli fermentation, namely: the simulated annealing (SA) algorithm, evolutionary algorithm (EA) and the differential evolution (DA) algorithm. SA parameterization consisted of 20000 iterations, population size of 50 and the interpolation factor was 1000. EA parameterization consisted of 2000 iterations, population size of 200 and the interpolation factor was 1000 . DA parameterization consisted of 10000 iterations, population size of 20 and the interpolation factor was 1000. Tests were carried out on packs of six simultaneous instantiations, for each algorithm, simulating a fermentation process of $25 \mathrm{~h}$, needing a processing time of about 6 hours to complete them. The results showing the optimal feed values produced by each one of the referred algorithms are depicted, respectively, in Fig.1, Fig.2 and Fig.3. While the results obtained with SA and EA showed concise values with marginal variations, the results provided by the differential evolution algorithm (see Fig. 3) were completely homogeneous considering the six simulations.

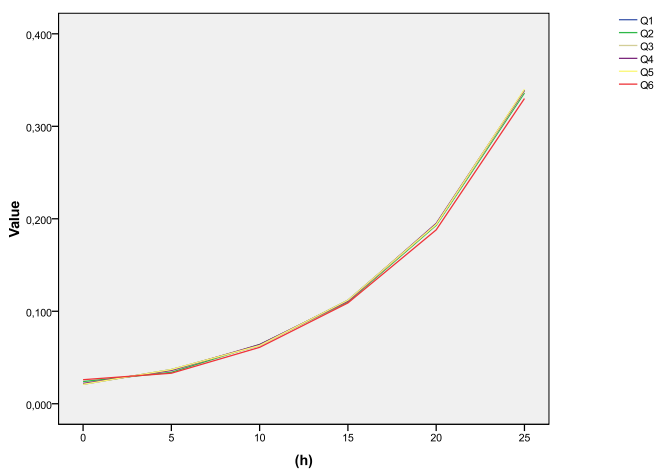

Fig. 1. Results obtained using the simulated annealing algorithm.

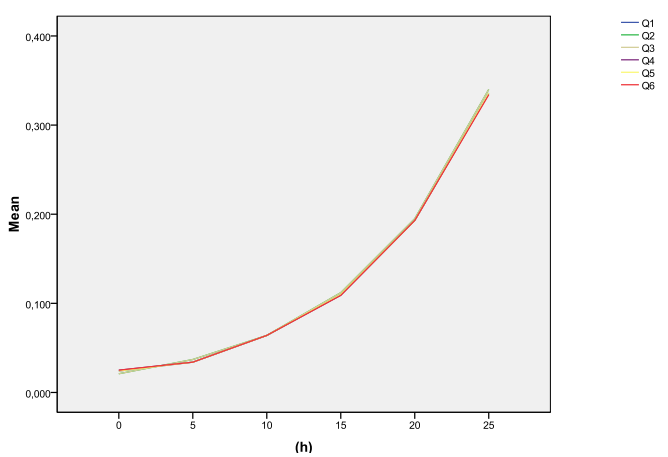

Fig. 2. Results obtained using the evolutionary algorithm. 


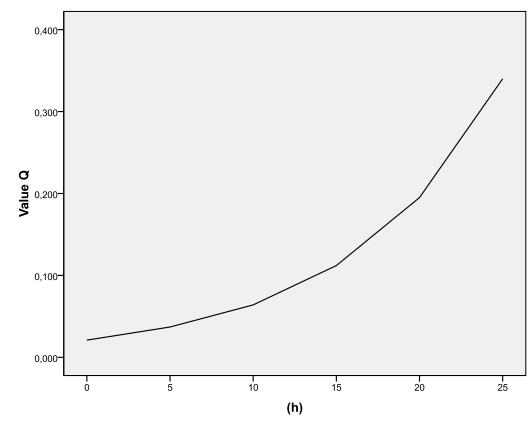

Fig. 3. Results obtained using the differential evolution algorithm.

The results for the objective function $J$, described in the Eq. (1), were also very similar. $J=9.222$ for the $\mathrm{SA} ;=9.26$ for the DA, and $J=9.123$ for the EA.

\section{Conclusions}

Optferm achieves optimum feed profiles to increase production in the escherichia coli fermentation process. The results obtained are consistent among the methods we used, as well as among the instantiations of the simulations of each algorithm we ran. The public domain tool is user-friendly, platform-independent, modular and open-source. Optferm is work in progress and we expect, in future versions, a better interface to construct new models, instead of coding them directly in Java, and additionally, better visualization capabilities to display the results.

\section{References}

[1] R. Mendes, I. Rocha, E. Ferreira, e M. Rocha, "A Comparison of Algorithms for the Optimization of Fermentation Processes," in 2006 IEEE International Conference on Evolutionary Computation, pp. 2018-2025.

[2] U. Yüzgeç, M. Türker, e A. Hocalar, "On-line evolutionary optimization of an industrial fed-batch yeast fermentation process," ISA Transactions, vol. 48, n. 1, pp. 79-92, Jan. 2009.

[3] B. Teusink, A. Wiersma, L. Jacobs, R. A. Notebaart, e E. J. Smid, "Understanding the adaptive growth strategy of Lactobacillus plantarum by in silico optimisation," PLoS Computational Biology, vol. 5, n. 6, p. e1000410, Jun. 2009.

[4] A. Pinto Mariano et al., "Optimization strategies based on sequential quadratic programming applied for a fermentation process for butanol production," Applied Biochemistry and Biotechnology, vol. 159, n. 2, pp. 366-381, Nov. 2009.

[5] J. M. Kavanagh e G. W. Barton, "Productivity improvement of recombinant Escherichia coli fermentation via robust optimization," Bioprocess and Biosystems Engineering, vol. 31, n. 2, pp. 137-143, Fev. 2008.

[6] I. Rocha, E. Ferreira, A. Veloso, J. Neves, e M. Rocha, "Evolutionary Algorithms for Static and Dynamic Optimization of Fed-batch Fermentation Processes," in Adaptive and Natural Computing Algorithms, Springer-Verlag, 2005, pp. 288-291.

[7] O. Rocha, P. Maia, I. Rocha, e M. Rocha, "OPTFERM-A Computational Platform for the Optimization of Fermentation Processes," presented at the European Simulation and Modelling Conference (ESM 2009), Leicester, UK, pp. 5-9, 2009. 